\title{
EXISTENCE RESULTS FOR SOME INITIAL AND BOUNDARY VALUE PROBLEMS WITHOUT GROWTH RESTRICTION
}

\author{
MARLENE FRIGON AND DONAL O'REGAN
}

(Communicated by Hal L. Smith)

\begin{abstract}
In this paper, using the Schauder Fixed Point Theorem, we establish some existence results or initial and boundary value problems for differential equations withouth growth restriction on the right member.
\end{abstract}

\section{INTRODUCTION}

In this paper, we give existence results for initial and boundary value problems without growth restriction on the right member. The paper is divided into two sections. In $\S 2$ we examine second-order problems of the form:

$$
\begin{aligned}
& \left(p y^{\prime}\right)^{\prime}(t)=f\left(t, y(t), p(t) y^{\prime}(t)\right) \quad \text { a.e. } t \in[0,1], \\
& a_{0} y(0)-b_{0} \lim _{t \rightarrow 0} p(t) y^{\prime}(t)=r_{0}, \\
& a_{1} y(1)+b_{1} \lim _{t \rightarrow 1} p(t) y^{\prime}(t)=r_{1},
\end{aligned}
$$

where $p \in C[0,1] \cap C^{1}(0,1)$ with $p(t)>0$ on $t \in(0,1)$, and $f:[0,1] \times$ $\mathbb{R}^{2} \rightarrow \mathbb{R}$ is a Carathéodory function. By a solution to $(B)$, we mean a function $y \in C[0,1] \cap C^{1}(0,1)$ such that $p y^{\prime}$ is absolutely continuous on $[0,1]$, and which satisfies the differential equation and boundary conditions. Boundary value problems of the form (B) have been extensively treated in the literature. In most of these papers, $f$ satisfies a growth condition in $y^{\prime}$. The results presented in this section are obtained without any growth assumption on $f$, and generalize some results deduced from the location of the zeros of the nonlinearity; see, for example, $[3,5,6,9]$. They rely on the notion of upper and lower surfaces for (B), that is, surfaces having a particular form and on which the function $f$ has a given sign. This notion was introduced in [3] in the particular case where $p \equiv 1$.

In $\S 3$ we examine the existence of solutions for a first-order initial value

Received by the editors April 22, 1993.

1991 Mathematics Subject Classification. Primary 34B15.

Reaserch supported in part by NSREC-Canada. 
problem of the form:

$$
\begin{aligned}
& y^{\prime}(t)=f(t, y(t)) \text { a.e. } t \in[0, T], \\
& y(0)=r
\end{aligned}
$$

where $f:[0, T] \times \mathbb{R} \rightarrow \mathbb{R}$ is a Carathéodory function. Here, by a solution to (I), we mean an absolutely continuous function on $[0, T]$ which satisfies the differential equation and the initial condition. The existence theorems presented here generalize some known results based on the notion of upper and lower solutions for the initial value problem; see $[1,6,8]$. In this paper, we prove the existence of a solution for the problem (I) between two functions $s_{1}$ and $s_{0}$ which are not necessarily absolutely continuous or continuous, without using the derivative or the Dini derivates of those functions.

All our results are obtained via the Schauder Fixed Point Theorem.

First of all, let us establish some notation. Let $p \in C[0,1] \cap C^{1}(0,1)$ be such that $p(t)>0$ on $t \in(0,1)$. We define the Banach space $K^{1}[0,1]$ by $\{y \in$ $\left.C[0,1] \cap C^{1}(0,1) \mid p y^{\prime} \in C[0,1]\right\}$ with the norm $\|y\|_{1}=\max \left\{\|y\|_{0},\left\|p y^{\prime}\right\|_{0}\right\}$ where $\|y\|_{0}=\max \{|y(t)| \mid t \in[0,1]\}$, and we define $K^{2}[0,1]$ by $\left\{y \in K^{1}[0,1] \mid\right.$ $\left.p y^{\prime} \in W^{1,1}[0,1]\right\}$. We denote $K_{b}^{1}[0,1]=\left\{y \in K^{1}[0,1] \mid y\right.$ satisfies boundary conditions $\left.a_{0} y(0)-b_{0} \lim _{t \rightarrow 0} p(t) y^{\prime}(t)=r_{0}, a_{1} y(1)+b_{1} \lim _{t \rightarrow 1} p(t) y^{\prime}(t)=r_{1}\right\}$, $K_{b}^{2}[0,1]=K^{2}[0,1] \cap K_{b}^{1}[0,1] ;$ and $C_{r}[0,1]=\{y \in C[0,1] \mid y(0)=r\}$.

For the sake of completeness, we give the following two results which will be used later. The second one is a Maximum Principle.

Lemma 1.1. Let $x:[0,1] \rightarrow \mathbb{R}$ be an absolutely continuous function, and $U \subset$ $[0,1]$ be a measurable set such that $x(U)$ is negligeable. Then $x^{\prime}(t)=0$ a.e. $t \in U$.

Lemma 1.2. Let $p \in C[c, d] \cap C^{1}(c, d)$ be such that $p(t)>0$ for all $t \in$ $(c, d)$; and let $y \in K^{2}[c, d]$ be such that $\left(p y^{\prime}\right)^{\prime}(t) \geq 0$ a.e. $t \in(c, d)$, $a_{0} y(c)-b_{0} \lim _{t \rightarrow c} p(t) y^{\prime}(t) \leq 0$, and $a_{1} y(d)+b_{1} \lim _{t \rightarrow d} p(t) y^{\prime}(t) \leq 0$, where $\max \left\{a_{0}, a_{1}\right\}>0, a_{i}, b_{i} \geq 0, \max \left\{a_{i}, b_{i}\right\}>0, i=0,1$. Then $y(t) \leq 0$ for all $t \in[c, d]$.

Proof. Define

$$
G(t)= \begin{cases}y(t)^{2} & \text { if } y(t)>0, \\ 0 & \text { otherwise. }\end{cases}
$$

Then $\left(p y^{\prime}\right)^{\prime}(t) G(t) \geq 0$ a.e. $t \in(c, d)$. Integrating from $c$ to $d$ and using the integration by parts formula gives:

$$
G(d) \lim _{t \rightarrow d} p(t) y^{\prime}(t)-G(c) \lim _{t \rightarrow c} p(t) y^{\prime}(t)-\int_{c}^{d} p(t) y^{\prime}(t) G^{\prime}(t) d t \geq 0 .
$$

We deduce that $y(t) \leq 0$ for all $t \in[c, d]$.

\section{BOUNDARY VALUE PROBLEMS}

In this section, we consider the boundary value problem (B) where $a_{i}, b_{i} \geq$ $0, \max \left\{a_{i}, b_{i}\right\}>0, i=0,1, \max \left\{a_{0}, a_{1}\right\}>0$, and $p$ and $f$ satisfy the following assumption:

(H) $p \in C[0,1] \cap C^{1}(0,1), p(t)>0$ for all $t \in(0,1), f:[0,1] \times \mathbb{R}^{2} \rightarrow \mathbb{R}$ is a Carathéodory function, that is: (i) $t \mapsto f(t, y, q)$ is measurable for all 
$(y, q) \in \mathbb{R}^{2}$; (ii) $(y, q) \mapsto f(t, y, q)$ is continuous for a.e. $t \in[0,1]$; (iii) for any $r>0$, there exists $h_{r} \in L^{1}[0,1]$ such that $|f(t, y, q)| \leq h_{r}(t)$ a.e. $t \in[0,1]$, and for all $|y| \leq r,|q| \leq r$. Moreover, $\int_{0}^{1} p^{-1}(s) \int_{0}^{s} h_{r}(x) d x d s<\infty$ if $a_{1} \neq 0$, and $\int_{0}^{1} p^{-1}(s) \int_{s}^{1} h_{r}(x) d x d s<\infty$ if $a_{1}=0$; and $p^{-1} \in L^{1}[0,1]$ if $a_{0}=r_{0}=0$ or $a_{1}=r_{1}=0$ do not occur.

We start with a particular case in order to illustrate arguments which will be used in a more general result (Theorem 2.8).

Consider the problem

$$
\begin{aligned}
& \left(p y^{\prime}\right)^{\prime}(t)=f\left(t, y(t), p(t) y^{\prime}(t)\right) \quad \text { a.e. } t \in[0,1], \\
& \lim _{t \rightarrow 0} p(t) y^{\prime}(t)=0 \\
& y(1)+b \lim _{t \rightarrow 1} p(t) y^{\prime}(t)=0
\end{aligned}
$$

where $b \geq 0$.

Theorem 2.1. Assume there exist $M_{0} \geq 0 \geq M_{1}$ such that $f\left(t, M_{0}, 0\right) \geq$ $0 \geq f\left(t, M_{1}, 0\right)$ a.e. $t \in[0,1]$ and there exist two functions $s_{0}:[0,1] \times$ $\left[M_{1}, M_{0}\right] \rightarrow[0, \infty), s_{1}:[0,1] \times\left[M_{1}, M_{0}\right] \rightarrow(-\infty, 0]$ such that:

(1) $x \mapsto s_{i}(t, x)$ is continuous for a.e. $t \in[0,1], i=0,1$;

(2) $s_{0}\left(t_{1}, x_{1}\right) \leq s_{0}\left(t_{2}, x_{2}\right)$ for all $t_{1} \leq t_{2}, x_{1} \leq x_{2}$; and $s_{1}\left(t_{1}, x_{1}\right) \leq$ $s_{1}\left(t_{2}, x_{2}\right)$ for all $t_{1} \geq t_{2}, x_{1} \leq x_{2}$

(3) $f\left(t, x, s_{0}(t, x)\right) \leq 0 \leq f\left(t, x, s_{1}(t, x)\right)$ for a.e. $t \in[0,1]$ and all $x \in\left[M_{1}, M_{0}\right]$.

Then the problem (2.1) has a solution such that $M_{1} \leq y(t) \leq M_{0}, s_{1}(t, y(t)) \leq$ $p(t) y^{\prime}(t) \leq s_{0}(t, y(t))$ for all $t \in[0,1]$.

To prove this theorem, we modify the problem (2.1), and we show that this modified problem has a solution which is also a solution to (2.1).

Define the function $f_{1}:[0,1] \times \mathbb{R}^{2} \rightarrow \mathbb{R}$ by

$$
f_{1}(t, y, q)= \begin{cases}f\left(t, M_{0}, 0\right) & \text { if } y>M_{0} \\ f\left(t, M_{1}, 0\right) & \text { if } y<M_{1}, \\ f\left(t, y, s_{0}(t, y)\right) & \text { if } M_{1} \leq y \leq M_{0}, q>s_{0}(t, y) \\ f\left(t, y, s_{1}(t, y)\right) & \text { if } M_{1} \leq y \leq M_{0}, q<s_{1}(t, y) \\ f(t, y, q) & \text { if } M_{1} \leq y \leq M_{0}, s_{1}(t, y) \leq q \leq s_{0}(t, y)\end{cases}
$$

It is clear that this function may not be Carathéodory, but we have the following result:

Proposition 2.2. Under the assumptions of the previous theorem, the operator $N_{1}: K^{1}[0,1] \rightarrow C_{0}[0,1]$ defined by $N_{1}(y)(t)=\int_{0}^{t} f_{1}\left(\tau, y(\tau), p(\tau) y^{\prime}(\tau)\right) d \tau$ is well defined, continuous, and compact.

Proof. Let $R>\max \left\{\left|M_{0}\right|,\left|M_{1}\right|\right\}$ such that $\left|s_{i}(t, y)\right| \leq R$ for all $t \in[0,1]$ and $y \in\left[M_{1}, M_{0}\right]$; and let $h_{R} \in L^{1}[0,1]$ be the function given in (H)(iii). Then

$$
\left|f_{1}\left(t, y(t), p(t) y^{\prime}(t)\right)\right| \leq h_{R}(t) \text { a.e. } t \in[0,1] \text { and for all. } y \in K^{1}[0,1] .
$$


So, $N_{1}$ is well defined. Now, assume that $y_{n} \rightarrow y$ in $K^{1}[0,1]$. By the inequality (2.2) and the Lebesgue Convergence Dominated Theorem, it suffices to show that

$$
f_{1}\left(t, y_{n}(t), p(t) y_{n}^{\prime}(t)\right) \rightarrow f_{1}\left(t, y(t), p(t) y^{\prime}(t)\right) \quad \text { a.e. } t \in[0,1]
$$

to get that $N_{1} y_{n} \rightarrow N_{1} y$ in $C_{0}[0,1]$.

It is clear that $(2.3)$ holds a.e. on $\left\{t \in[0,1] \mid y(t) \neq M_{0}\right.$, and $\left.y(t) \neq M_{1}\right\}$. On the other hand, by Lemma $1.1, y^{\prime}(t)=0$ a.e. on $\{t \in[0,1] \mid y(t)=$ $M_{0}$, or $\left.y(t)=M_{1}\right\}$. So, $s_{1}(t, y(t)) \leq p(t) y^{\prime}(t)=0 \leq s_{0}(t, y(t))$ a.e. on that set. The assumptions (1) and (H)(ii) imply that (2.3) holds a.e. on that set.

The compactness of $N_{1}$ follows from (2.2) and the Arzela-Ascoli Theorem.

We consider the modified problem

$$
\begin{aligned}
& \left(p y^{\prime}\right)^{\prime}(t)=f_{1}\left(t, y(t), p(t) y^{\prime}(t)\right) \quad \text { a.e. } t \in[0,1] \\
& \lim _{t \rightarrow 0} p(t) y^{\prime}(t)=0 \\
& y(1)+b \lim _{t \rightarrow 1} p(t) y^{\prime}(t)=0 .
\end{aligned}
$$

Proposition 2.3. Under the assumptions of Theorem 2.1, problem (2.4) has a solution.

Proof. Set $C_{0}^{R}=\left\{g \in C_{0}[0,1]|| g(t) \mid \leq \int_{0}^{t} h_{R}(s) d s\right\}$ where $h_{R}$ is the function satisfying inequality (2.2). Define the continuous operator $L^{-1}: C_{0}^{R} \rightarrow K_{b}^{1}$ by

$$
L^{-1}(g)(t)=-b g(1)-\int_{t}^{1} g(s) p^{-1}(s) d s .
$$

From (2.2), $N_{1}(y) \in C_{0}^{R}$ for all $y \in K_{b}^{1}$. Remark that $y$ is a solution to (2.4) if and only if $y$ is a fixed point of $L^{-1} \circ N_{1}: K_{b}^{1} \rightarrow K_{b}^{1}$ (see [4] for details). By Proposition 2.2, the operator $L^{-1} \circ N_{1}$ is compact. The Schauder Fixed Point Theorem gives the existence of a fixed point to this operator, and hence a solution to $(2.4)$.

Proof of Theorem 2.1. Let $y$ be a solution to (2.4) given by the previous proposition. We want to show that $y$ is a solution to (2.1).

We claim that $M_{1} \leq y(t) \leq M_{0}$. Indeed, let $u(t)=y(t)-M_{0}$. By assumption and the definition of $f_{1}$, we have

$$
\left(p u^{\prime}\right)^{\prime}(t) \geq 0 \text { a.e. on }\{t \mid u(t)>0\} \text {. }
$$

Boundary conditions and Lemma 1.2 imply that $y(t) \leq M_{0}$ for all $t \in[0,1]$. Similarly we get the other inequality.

On the other hand, assume there exists $t_{1} \in[0,1]$ such that $p\left(t_{1}\right) y^{\prime}\left(t_{1}\right)>$ $s_{0}\left(t_{1}, y\left(t_{1}\right)\right)$. Since $\lim _{t \rightarrow 0} p(t) y^{\prime}(t)=0 \leq s_{0}(0, y(0))$, there exists $t_{2}<t_{1}$ such that $s_{0}\left(t_{2}, y\left(t_{2}\right)\right) \geq p\left(t_{2}\right) y^{\prime}\left(t_{2}\right)$ and $s_{0}(t, y(t)) \leq p(t) y^{\prime}(t)$ for all $t \in\left(t_{2}, t_{1}\right)$. By assumption (2) and the definition of $f_{1}$, we get

$$
0<p\left(t_{1}\right) y^{\prime}\left(t_{1}\right)-p\left(t_{2}\right) y^{\prime}\left(t_{2}\right)=\int_{t_{2}}^{t_{1}}\left(p y^{\prime}\right)^{\prime}(t) d t \leq 0,
$$

a contradiction. The other inequality is obtained in the same way. 
Therefore, the solution $y$ is such that $M_{1} \leq y(t) \leq M_{0}$ and $s_{1}(t, y(t)) \leq$ $p(t) y^{\prime}(t) \leq s_{0}(t, y(t))$ for all $t \in[0,1]$; and consequently, $y$ is a solution to (2.1).

Now, we give a more general result. First of all, we need to introduce some definitions. The notion of upper and lower surfaces was introduced in [3] in the particular case where $p \equiv 1$.

Definition 2.4. A function $\alpha \in K^{2}[0,1]$ (resp. $\beta \in K^{2}[0,1]$ ) is called a lower solution (resp. upper solution) to (B) if $\left(p \alpha^{\prime}\right)^{\prime}(t) \geq f\left(t, \alpha(t), p(t) \alpha^{\prime}(t)\right)$ a.e $t \in$ $[0,1] ; a_{0} \alpha(0)-b_{0} \lim _{t \rightarrow 0} p(t) \alpha^{\prime}(t) \leq r_{0}, a_{1} \alpha(1)+b_{1} \lim _{t \rightarrow 1} p(t) \alpha^{\prime}(t) \leq r_{1}$ (resp. $\left(p \beta^{\prime}\right)^{\prime}(t) \leq f\left(t, \beta(t), p(t) \beta^{\prime}(t)\right)$ a.e $t \in[0,1] ; a_{0} \beta(0)-b_{0} \lim _{t \rightarrow 0} p(t) \beta^{\prime}(t) \geq$ $\left.r_{0}, a_{1} \beta(1)+b_{1} \lim _{t \rightarrow 1} p(t) \beta^{\prime}(t) \geq r_{1}\right)$.

Let $\alpha \leq \beta$ in $C[0,1]$ and $D=\{(t, y) \in[0,1] \times \mathbb{R} \mid \alpha(t) \leq y \leq \beta(t)\}$.

Definition 2.5. A surface $S \subset D \times \mathbb{R}$ is an upper surface to $D$ for (B) if there exist two functions $s: D \rightarrow[0, \infty), c \in K^{1}[0,1]$ such that $S=$ $\{(t, x, s(t, x)) \mid(t, x) \in D\}, \quad \alpha \leq c \leq \beta$, and satisfying the following conditions:

(i) $(x-c(t)) f(t, x, s(t, x)) \geq 0$ a.e. $t \in[0,1],(t, x) \in D$;

(ii) there exist $N \subset[0,1]$ and $E \subset \mathbb{R}$, two negligeable sets such that the function $x \mapsto s(t, x)$ is continuous for all $(t, x) \in D \backslash(N \times E)$;

(iii) $p(t) c^{\prime}(t) \leq s(t, c(t))$ for all $t \in[0,1]$;

(iv) $s\left(t_{1}, x_{1}\right) \geq s\left(t_{2}, x_{2}\right)$ (resp. $s\left(t_{1}, x_{1}\right) \leq s\left(t_{2}, x_{2}\right)$ ) for all $t_{1} \leq t_{2}$, $x_{1} \leq x_{2}$ such that $\left(t_{1}, x_{1}\right)$ and $\left(t_{2}, x_{2}\right)$ are in the same connected part of $D^{+}=\overline{\{(t, x) \mid x>c(t)\}}$ (resp. $D^{-}=\overline{\{(t, x) \mid x<c(t)\}}$ );

(v) $a_{0} c(0) \leq r_{0}+b_{0} s(0, \alpha(0)), a_{1} c(1) \geq r_{1}-b_{1} s(1, \beta(1))$.

Definition 2.6. A surface $S \subset D \times \mathbb{R}$ is a lower surface to $D$ for (B) if there exist two functions $s: D \rightarrow(-\infty, 0], c \in K^{1}[0,1]$ such that $S=\{(t, x, s(t, x))$ $(t, x) \in D\}, \quad \alpha \leq c \leq \beta$, and satisfying (i), (ii) of Definition 2.5 and the following conditions:

(iii) $p(t) c^{\prime}(t) \geq s(t, c(t))$ for all $t \in[0,1]$;

(iv) $s\left(t_{1}, x_{1}\right) \leq s\left(t_{2}, x_{2}\right)$ (resp. $s\left(t_{1}, x_{1}\right) \geq s\left(t_{2}, x_{2}\right)$ ) for all $t_{1} \geq t_{2}$, $x_{1} \leq x_{2}$ such that $\left(t_{1}, x_{1}\right)$ and $\left(t_{2}, x_{2}\right)$ are in the same connected part of $D^{+}=\overline{\{(t, x) \mid x>c(t)\}}$ (resp. $D^{-}=\overline{\{(t, x) \mid x<c(t)\}}$ );

(v) $a_{0} c(0) \geq r_{0}+b_{0} s(0, \beta(0)), \quad a_{1} c(1) \leq r_{1}-b_{1} s(1, \alpha(1))$.

Remark 2.7. If $f$ is a Carathéodory function and $S$ is an upper (resp. lower) surface to $D$ for (B), without loss of generality, we can assume that

(1) $S$ is bounded;

(2) for $y=\alpha(t), \beta(t)$, and $c(t), \quad s(t, y)=\limsup _{(t, x) \in D, x \rightarrow y} s(t, x)$ (resp. $\left.s(t, y)=\liminf _{(t, x) \in D, x \rightarrow y} s(t, x)\right)$.

Theorem 2.8. Let $p, f$ be functions satisfying $(\mathrm{H})$. Assume there exist $\alpha \leq \beta$, respectively, lower and upper solutions to (B). Set $D=\{(t, y) \in[0,1] \times \mathbb{R} \mid \alpha(t) \leq$ $y \leq \beta(t)\}$, and suppose there exist $S_{1}=S_{1}\left(s_{1}, c_{1}\right)$ and $S_{0}=S_{0}\left(s_{0}, c_{0}\right)$, respectively, lower and upper surfaces to $D$ for $(\mathrm{B})$. Then the problem (B) has a 
solution such that $\alpha(t) \leq y(t) \leq \beta(t), s_{1}(t, y(t)) \leq p(t) y^{\prime}(t) \leq s_{0}(t, y(t))$ for all $t \in[0,1]$.

To $(t, y, q) \in D \times \mathbb{R}$ we associate $\tilde{q}$ defined by

$$
\tilde{q}= \begin{cases}s_{0}(t, y) & \text { if } q>s_{0}(t, y), \\ q & \text { if } s_{1}(t, y) \leq q \leq s_{0}(t, y), \\ s_{1}(t, y) & \text { if } q<s_{1}(t, y) .\end{cases}
$$

Lemma 2.9. Under the assumptions of Theorem 2.8, there exist four measurable functions $\varepsilon_{i, \alpha}, \varepsilon_{i, \beta}:[0,1] \rightarrow[0, \infty)$ such that

$$
\begin{aligned}
& \begin{cases}\alpha(t)<\alpha(t)+\varepsilon_{0, \alpha}(t)<c_{0}(t) & \text { if } \alpha(t)<c_{0}(t), s_{0}(t, \alpha(t))<p(t) \alpha^{\prime}(t), \\
\varepsilon_{0, \alpha}(t)=0 & \text { otherwise; }\end{cases} \\
& \begin{cases}\beta(t)>\beta(t)-\varepsilon_{0, \beta}(t)>c_{0}(t) & \text { if } \beta(t)>c_{0}(t), s_{0}(t, \beta(t))<p(t) \beta^{\prime}(t), \\
\varepsilon_{0, \beta}(t)=0 & \text { otherwise; }\end{cases}
\end{aligned}
$$

moreover, $q>s_{0}(t, y)$ for all $(y, q) \in B_{0, \alpha}(t) \cup B_{0, \beta}(t)$. Similarly for $i=1$, where

$$
\begin{aligned}
& B_{i, \alpha}(t)= \begin{cases}\{(y, q) \mid(t, y) \in D, \\
\left.\left\|(y, q)-\left(\alpha(t), p(t) \alpha^{\prime}(t)\right)\right\|<\varepsilon_{i, \alpha}(t)\right\} & \text { if } \varepsilon_{i, \alpha}(t) \neq 0, \\
\varnothing & \text { otherwise }\end{cases} \\
& B_{i, \beta}(t)= \begin{cases}\{(y, q) \mid(t, y) \in D, & \text { if } \varepsilon_{i, \beta}(t) \neq 0, \\
\left.\left\|(y, q)-\left(\beta(t), p(t) \beta^{\prime}(t)\right)\right\|<\varepsilon_{i, \beta}(t)\right\} & \text { otherwise. }\end{cases}
\end{aligned}
$$

Proof. Let

$$
\begin{aligned}
A_{i}(t) & =\overline{\operatorname{co}}\left\{\left(y, s_{i}(t, y)\right) \mid(t, y) \in D\right\}, \\
d_{i, \alpha}(t) & =\operatorname{dist}\left(\left(\alpha(t), p(t) \alpha^{\prime}(t)\right), A_{i}(t)\right) .
\end{aligned}
$$

Choose $\varepsilon_{i, \alpha}(t) \leq \min \left\{c_{i}(t)-\alpha(t), d_{i, \alpha}(t)\right\} / 2$. Remark that if $p\left(\widetilde{t) \alpha^{\prime}}(t) \neq\right.$ $p(t) \alpha^{\prime}(t)$ and $c_{i}(t)-\alpha(t)>0$, then $d_{i, \alpha}(t)>0$ by Definitions 2.5 and 2.6 and Remark 2.7(2). We define $\varepsilon_{i, \beta}$ similarly.

Define the function $f_{2}:[0,1] \times \mathbb{R}^{2} \rightarrow \mathbb{R}$ by

$$
f_{2}(t, y, q)=\left\{\begin{array}{cl}
\max \left\{f\left(t, \beta(t), p(t) \beta^{\prime}(t)\right), f\left(t, \beta(t), p\left(\widetilde{t) \beta^{\prime}}(t)\right)\right\}\right. & \text { if } y>\beta(t), \\
\min \left\{f\left(t, \alpha(t), p(t) \alpha^{\prime}(t)\right), f\left(t, \alpha(t), p\left(\widetilde{t) \alpha^{\prime}}(t)\right)\right\}\right. & \text { if } y<\alpha(t), \\
\left(1-\lambda_{i, \beta}(t, y, q)\right) \max \{f(t, y, q), f(t, y, \widetilde{q})\} & \\
\quad+\lambda_{i, \beta}(t, y, q) f(t, y, \widetilde{q}) & \text { if }(y, q) \in B_{i, \beta}(t), i=0,1, \\
\left(1-\lambda_{i, \alpha}(t, y, q)\right) \min \{f(t, y, q), f(t, y, \widetilde{q})\} & \\
\quad+\lambda_{i, \alpha}(t, y, q) f(t, y, \widetilde{q}) & \text { if }(y, q) \in B_{i, \alpha}(t), i=0,1, \\
f(t, y, \widetilde{q}) & \text { otherwise. }
\end{array}\right.
$$

where $\lambda_{i, \beta}(t, y, q)=\left(\varepsilon_{i, \beta}(t)\right)^{-1}\left\|(y, q)-\left(\beta(t), p(t) \beta^{\prime}(t)\right)\right\| \quad$ if $\quad(y, q) \in$ $B_{i, \beta}(t)$, and $\lambda_{i, \alpha}(t, y, q)=\left(\varepsilon_{i, \alpha}(t)\right)^{-1}\left\|(y, q)-\left(\alpha(t), p(t) \alpha^{\prime}(t)\right)\right\| \quad$ if $(y, q) \in$ $B_{i, \alpha}(t), i=0,1$. 
Remark 2.10. On $\left\{(t, y, q) \in[0,1] \times \mathbb{R}^{2} \mid \alpha(t) \leq y \leq \beta(t), s_{1}(t, y) \leq q \leq\right.$ $\left.s_{0}(t, y)\right\}, f_{2}(t, y, q)=f(t, y, q)$.

For the sake of brevity, we assume that $a_{1} \neq 0$; the proof of Theorem 2.8 is similar for $a_{1}=0$. Without loss of generality, fix $a_{1}=1$.

Proposition 2.11. Under the assumptions of Theorem 2.8, the operator $\mathrm{N}_{2}$ : $K^{1}[0,1] \rightarrow C_{0}[0,1]$ defined by

$$
N_{2}(y)(t)=\int_{0}^{t} f_{2}\left(\tau, y(\tau), p(\tau) y^{\prime}(\tau)\right) d \tau
$$

is well defined, continuous, and compact.

Proof. Let $R>\max \left\{\|\alpha\|_{0},\|\beta\|_{0},\left\|p \alpha^{\prime}\right\|_{0},\left\|p \beta^{\prime}\right\|_{0}\right\}$ such that $\left|s_{i}(t, y)\right| \leq R$ for all $(t, y) \in D$; and let $h_{R} \in L^{1}[0,1]$ be the function given in $(\mathrm{H})(\mathrm{iii})$. We have

$$
\left|f_{2}\left(t, y(t), p(t) y^{\prime}(t)\right)\right| \leq h_{R}(t) \quad \text { a.e. } t \in[0,1] \text { and all } y \in K^{1}[0,1] \text {. }
$$

Hence, $N_{2}$ is well defined and the compactness of $N_{2}$ follows from (2.5) and the Arzela-Ascoli Theorem.

To show that $N_{2}$ is continuous, according to the inequality (2.5) and the Lebesgue Dominated Convergence Theorem, it suffices to show that

$$
f_{2}\left(t, y_{n}(t), p(t) y_{n}^{\prime}(t)\right) \rightarrow f_{2}\left(t, y(t), p(t) y^{\prime}(t)\right) \text { a.e. } t \in[0,1]
$$

when $y_{n} \rightarrow y$ in $K^{1}[0,1]$.

It is clear that the relation (2.6) holds a.e. on $\{t \mid \alpha(t) \neq y(t)$ and $\beta(t) \neq y(t)\}$. On the other hand, on $\left\{t \mid \alpha(t)=y(t), B_{i, \alpha}(t) \neq \varnothing\right\}, p(t) \alpha^{\prime}(t)=p(t) y^{\prime}(t)$ a.e. and

$$
f_{2}\left(t, y(t), p(t) y^{\prime}(t)\right)=\min \left\{f\left(t, \alpha(t), p(t) \alpha^{\prime}(t)\right), f\left(t, \alpha(t), p\left(\widetilde{t) \alpha^{\prime}}(t)\right)\right\} .\right.
$$

If $y_{n}(t) \geq \alpha(t)$, then for $n$ sufficiently large, we have $\left(y_{n}(t), p(t) y_{n}^{\prime}(t)\right) \in$ $B_{i, \alpha}(t)$ and $\lambda_{i, \alpha}\left(t, y_{n}(t), p(t) y_{n}^{\prime}(t)\right) \rightarrow 0$. Hence (2.6) holds a.e. on that set. On $\left\{t \mid \alpha(t)=y(t), \quad B_{0, \alpha}(t)=B_{1, \alpha}(t)=\varnothing\right\}, p\left(\widetilde{t) \alpha^{\prime}}(t)=p(t) \alpha^{\prime}(t)=p(t) y^{\prime}(t)\right.$ a.e. and (2.6) holds a.e. Similarly, the relation (2.6) holds a.e. on $\{t \mid \beta(t)=$ $y(t)\}$ and the proof is complete.

Consider the modified problem

$$
\begin{aligned}
& \left(p y^{\prime}\right)^{\prime}(t)=f_{2}\left(t, y(t), p(t) y^{\prime}(t)\right) \text { a.e. } t \in[0,1], \\
& a_{0} y(0)-b_{0} \lim _{t \rightarrow 0} p(t) y^{\prime}(t)=r_{0}, \\
& y(1)+b_{1} \lim _{t \rightarrow 1} p(t) y^{\prime}(t)=r_{1} .
\end{aligned}
$$

Proposition 2.12. Under the assumptions of Theorem 2.8, problem (2.7) has a solution.

Proof. To prove this result, argue as for Proposition 2.3 with

$$
L^{-1}(g)(t)= \begin{cases}r_{1}+r_{0} b_{1} b_{0}^{-1}-b_{1} g(1)+\int_{t}^{1}\left(r_{0} b_{0}^{-1}-g(s)\right) p^{-1}(s) d s & \text { if } a_{0}=0, \\ \left(r_{0}+b_{0} A\right) a_{0}^{-1}+\int_{0}^{t}(A+g(s)) p^{-1}(s) d s & \text { if } a_{0} \neq 0\end{cases}
$$

where

$$
A=\frac{r_{1}-r_{0} a_{0}^{-1}-b_{1} g(1)-\int_{0}^{1} g(s) p^{-1}(s) d s}{b_{1}+b_{0} a_{0}^{-1}+\int_{0}^{1} p^{-1}(s) d s}
$$


Proof of Theorem 2.8. Let $y$ be a solution to (2.7) given by Proposition 2.12. According to Remark 2.10, we must show that $\alpha(t) \leq y(t) \leq \beta(t), s_{1}(t, y(t)) \leq$ $p(t) y^{\prime}(t) \leq s_{0}(t, y(t))$ for all $t \in[0,1]$.

By assumption and the definition of $f_{2}$, we have

$$
\left(p y^{\prime}\right)^{\prime}(t) \geq\left(p \beta^{\prime}\right)^{\prime}(t) \text { a.e. on }\{t \mid y(t)>\beta(t)\}
$$

and

$$
\left(p y^{\prime}\right)^{\prime}(t) \leq\left(p \alpha^{\prime}\right)^{\prime}(t) \text { a.e. on }\{t \mid y(t)>\alpha(t)\}
$$

By boundary conditions and the Maximum Principle, Lemma 1.2, we deduce that $\alpha(t) \leq y(t) \leq \beta(t)$.

On the other hand, assume that $p(t) y^{\prime}(t) \not \leq s_{0}(t, y(t))$. By Definition 2.5 and boundary conditions, one of the following cases holds:

(a) there exist $t_{1}<t_{2} \in[0,1]$ such that $y(t) \geq c_{0}(t), p(t) y^{\prime}(t) \geq s_{0}(t, y(t))$ a.e. $t \in\left(t_{1}, t_{2}\right), p\left(t_{1}\right) y^{\prime}\left(t_{1}\right)>s_{0}\left(t_{1}, y\left(t_{1}\right)\right), p\left(t_{2}\right) y^{\prime}\left(t_{2}\right) \leq s_{0}\left(t_{2}, y\left(t_{2}\right)\right)$;

(b) there exist $t_{1}<t_{2} \in[0,1]$ such that $y(t) \leq c_{0}(t), p(t) y^{\prime}(t) \geq s_{0}(t, y(t))$ a.e. $t \in\left(t_{1}, t_{2}\right), p\left(t_{1}\right) y^{\prime}\left(t_{1}\right) \leq s_{0}\left(t_{1}, y\left(t_{1}\right)\right), p\left(t_{2}\right) y^{\prime}\left(t_{2}\right)>s_{0}\left(t_{2}, y\left(t_{2}\right)\right)$. Without loss of generality, assume (a) holds. Definition 2.5(i) and the definition of $f_{2}$ imply that $\left(p y^{\prime}\right)^{\prime}(t) \geq 0$ a.e. $t \in\left(t_{1}, t_{2}\right)$. Therefore, using the monotonicity condition on $s_{0}$ (Definition $2.5($ iv) ), we get

$$
0>p\left(t_{2}\right) y^{\prime}\left(t_{2}\right)-p\left(t_{1}\right) y^{\prime}\left(t_{1}\right)=\int_{t_{1}}^{t_{2}}\left(p y^{\prime}\right)^{\prime}(t) d t \geq 0,
$$

a contradiction. Similarly, we can show that $s_{1}(t, y(t)) \leq p(t) y^{\prime}(t)$.

\section{INITIAL VALUE PROBLEMS}

In this section, we consider the initial value problem (I). The following theorem gives the existence of a solution to (I) under an assumption which generalizes the usual notion of upper and lower solutions for a first-order problem; see $[1,6,8]$.

Theorem 3.1. Let $f$ be a Carathéodory function. Assume there exist two functions $s_{0}, s_{1}$ such that:

(i) $s_{i}=b_{i}+c_{i}$ with $c_{i} \in C[0, T]$ and $b_{i}$ a function of bounded variation on $[0, T], i=0,1$;

(ii) $s_{1}\left(0^{+}\right) \leq r \leq s_{0}\left(0^{+}\right), s_{1}(t) \leq s_{0}(t)$ for all $t \in[0, T]$;

(iii) $\int_{a}^{b} f\left(t, s_{0}(t)\right) d t \leq s_{0}\left(b^{-}\right)-s_{0}\left(a^{+}\right), \quad \int_{a}^{b} f\left(t, s_{1}(t)\right) d t \geq s_{1}\left(b^{-}\right)-s_{1}\left(a^{+}\right)$ for all $a<b \in[0, T]$.

Then problem (I) has a solution such that $s_{1}(t) \leq y(t) \leq s_{0}(t)$ for a.e. $t \in[0, T]$.

Proof. Define $f_{3}:[0, T] \times \mathbb{R} \rightarrow \mathbb{R}$ by

$$
f_{3}(t, y)= \begin{cases}f\left(t, s_{0}(t)\right) & \text { if } y>s_{0}(t), \\ f(t, y) & \text { if } s_{1}(t) \leq y \leq s_{0}(t), \\ f\left(t, s_{1}(t)\right) & \text { if } y<s_{1}(t)\end{cases}
$$

and consider the associated problem

$$
\begin{aligned}
& y^{\prime}(t)=f_{3}(t, y(t)) \text { a.e. } t \in[0, T], \\
& y(0)=r .
\end{aligned}
$$


A solution to (3.1) is a fixed point to the operator $N_{3}: C_{r}[0, T] \rightarrow C_{r}[0, T]$ defined by

$$
N_{3}(y)(t)=r+\int_{0}^{t} f_{3}(\tau, y(\tau)) d \tau .
$$

Observe that the operator $N_{3}$ is well defined since $f$ is Carathéodory and $s_{0}, s_{1}$ are measurable and bounded, and that there exists a function $h \in L^{1}[0, T]$ such that

$$
\left|f_{3}(t, y(t))\right| \leq h(t) \text { a.e. } t \in[0, T] \text { and all } y \in C_{r}[0, T] .
$$

This inequality and the Arzela-Ascoli Theorem imply that $N_{3}$ is compact. Moreover, the continuity of $N_{3}$ follows from the inequality (3.2), the Lebesgue Dominated Convergence Theorem, and the following relation:

$$
f_{3}\left(t, y_{n}(t)\right) \rightarrow f_{3}(t, y(t)) \text { a.e. } t \in[0, T]
$$

when $y_{n} \rightarrow y$ in $C_{r}[0, T]$.

Therefore, the Schauder Fixed Point Theorem gives the existence of a fixed point to $N_{3}$; that is, a solution to $y$ to $(3.1)$.

Now, we want to show that $y$ satisfies $s_{1}(t) \leq y(t) \leq s_{0}(t)$ for a.e. $t \in[0, T]$, and consequently, is a solution to $(\mathrm{I})$. Assume $y(t) \leq s_{0}(t)$ a.e. $t \in[0, T]$. Since $y(0)=r \leq s_{0}\left(0^{+}\right)$, there exist $t_{1}<t_{2} \in[0, T]$ such that $y\left(t_{1}\right) \leq s_{0}\left(t_{1}^{+}\right)$, $y\left(t_{2}\right)>s_{0}\left(t_{2}^{-}\right)$, and $y(t) \geq s_{0}(t)$ a.e. $t \in\left(t_{1}, t_{2}\right)$. By the definition of $f_{3}$ and assumption (iii), we have

$$
y\left(t_{2}\right)-y\left(t_{1}\right)=\int_{t_{1}}^{t_{2}} f\left(t, s_{0}(t)\right) d t \leq s_{0}\left(t_{2}^{-}\right)-s_{0}\left(t_{1}^{+}\right) .
$$

This leads to a contradiction. Similarly, we show the other inequality.

We get as a corollary a result concerning upper and lower solutions for a first-order problem; see [1, 8].

Corllary 3.2. Let $f$ be a Carathéodory function. Assume there exist two absolutely continuous functions $\alpha \leq \beta$ such that $\alpha(0) \leq r \leq \beta(0), f(t, \beta(t)) \leq$ $\beta^{\prime}(t), \alpha^{\prime}(t) \leq f(t, \alpha(t))$ a.e. $t \in[0, T]$. Then problem (I) has a solution such that $\alpha(t) \leq y(t) \leq \beta(t)$ for all $t \in[0, T]$.

Corollary 3.3. Let $f$ be a Carathéodory function. Assume there exist two nondecreasing functions $-s_{1}, s_{0}$ such that $s_{1} \leq s_{0}, s_{1}\left(0^{+}\right) \leq r \leq s_{0}\left(0^{+}\right)$, and $f\left(t, s_{0}(t)\right) \leq 0 \leq f\left(t, s_{1}(t)\right)$ a.e. $t \in[0, T]$. Then problem (I) has a solution such that $s_{1}(t) \leq y(t) \leq s_{0}(t)$ for all $t \in[0, T]$.

The next result generalizes a result of O'Farrell and O'Regan [7].

Corollary 3.4. Let $\psi, \phi:[0, T] \rightarrow[0, \infty)$ and $g: \mathbb{R} \rightarrow \mathbb{R}$ be continuous functions such that $\psi>0$ on $(0, T]$ and $\phi>0$ on $(0, T), \phi / \psi \in L^{1}[0, T]$. Assume there exist two constants $s_{1} \leq r \leq s_{0}$ such that $g\left(s_{1}\right) \geq 0 \geq g\left(s_{0}\right)$. Then the problem

$$
\begin{aligned}
& \psi(t) y^{\prime}(t)=\phi(t) g(y(t)) \text { a.e. } t \in[0, T], \\
& y(0)=r
\end{aligned}
$$

has a solution $y \in C[0, T] \cap C^{1}(0, T]$.

In Theorem 3.1, we can reverse the inequalities in assumption (iii) if assumption (ii) is replaced by a stronger one. 
Theorem 3.5. Let $f$ be a Carathéodory function. Assume there exist two functions $s_{0}, s_{1}$ such that:

(i) $s_{i}=b_{i}+c_{i}$ with $c_{i} \in C[0, T]$ and $b_{i}$ a function of bounded variation on $[0, T], i=0,1$;

(ii) $s_{1}\left(0^{+}\right)=r=s_{0}\left(0^{+}\right), s_{1}(t) \leq s_{0}(t)$ for all $t \in[0, T]$;

(iii) $\int_{a}^{b} f\left(t, s_{0}(t)\right) d t \geq s_{0}\left(b^{-}\right)-s_{0}\left(a^{+}\right), \quad \int_{a}^{b} f\left(t, s_{1}(t)\right) d t \leq s_{1}\left(b^{-}\right)-s_{1}\left(a^{+}\right)$ for all $a<b \in[0, T]$.

Then problem (I) has a solution such that $s_{1}(t) \leq y(t) \leq s_{0}(t)$ for a.e. $t \in[0, T]$. Proof. Let $\hat{r} \in\left[s_{1}\left(1^{-}\right), s_{0}\left(1^{-}\right)\right]$. Consider the problem

$$
\begin{aligned}
& y^{\prime}(t)=f(t, y(t)) \text { a.e. } t \in[0, T], \\
& y(1)=\hat{r} .
\end{aligned}
$$

By making the change of variable $\tau=1-t$, problem (3.3) can be written as a problem of form (I) on which we can apply Theorem 3.1. Therefore, problem (3.3) has a solution such that $s_{1}(t) \leq y(t) \leq s_{0}(t)$ for a.e. $t \in[0, T]$. By assumption (ii), we deduce that $y$ is a solution to (I).

\section{REFERENCES}

1. A. Cabada, The monotone method for first order problems with linear and nonlinear boundary conditions, preprint.

2. J. Dugundji and A. Granas, Fixed point theory, Vol. 1, PWN, Warszawa, 1982.

3. M. Frigon, Théorèmes d'existence pour des problèmes aux limites sans condition de croissance, Bull. Sci. Math. (2) 117 (1993), 377-400.

4. M. Frigon and D. O'Regan, Some general existence principles for ordinary differential equations, Topological Methods Nonlinear Anal. 2 (1993).

5. A. Granas, R. B. Guenther, and J. W. Lee, Nonlinear boundary value problems for ordinary differential equations, Dissertationes Math. 244 (1985), 1-128.

6. M. N. Nkashama, A generalized upper and lower solutions method and multiplicity results for nonlinear first order ordinary differential equations, J. Math. Anal. Appl. 140 (1989), 381-395.

7. A. G. O'Farrell and D. O'Regan, Existence results for some initial-and boundary-value problems, Proc. Amer. Math. Soc. 110 (1990), 661-673.

8. L. C. Piccinini, G. Stampacchia, and G. Vidossich, Ordinary differential equations in $\mathbb{R}^{n}$, Appl. Math. Sci., vol. 39, Springer-Verlag, New York, 1984.

9. A. Rodriguez and A. Tineo, Existence theorems for the Dirichlet problem without growth restrictions, J. Math. Anal. Appl. 135 (1988), 1-7.

Département de Mathematiques et Statistique, Universite de Montreal, C.P. 6128 , succ. A, Montréal, Canada H3C 3J7

E-mail address: frigon@ere.umontreal.ca

Department of Mathematics, University College Galway, Galway, Ireland

E-mail address: matoregan@bodkin.ucg.ie 